\title{
Electromyography Values of Chewing Muscles in Healthy and Bruxing Conditions
}

\author{
${ }^{1}$ Mikhail Soykher, ${ }^{2}$ Olga Orlova, ${ }^{2}$ Marina Soykher, ${ }^{2}$ Daria Shershneva, ${ }^{1}$ Lusine Vekilyan, ${ }^{1}$ Valery Kotlyarov, \\ ${ }^{1}$ Elizaveta Soykher, ${ }^{2}$ Anna Stroganova
}

\begin{abstract}
Introduction: Bruxism is one of the mounting questions in contemporary dentistry due to its high prevalence, various clinical presentations, difficulties in diagnostics and treatment, demanding a complex approach from doctors of different specialties.
\end{abstract}

Aim: The aim of the current study was to describe the electromyographic values of chewing muscles in healthy persons and patients with bruxism and to find the biomarkers for early diagnostics of bruxism.

Materials and methods: During retrospective investigation patients with bruxism were divided into two groups: group botox and bruxism. The control group was named the group-no complain.

Results: Remarkable differences were found in group botox (no complain) in tests, rest and pressing in occlusion for chewing muscles symmetry index (CMSI\%).

Conclusion: As a result, we revealed a total bio-electric muscle activity index (BMIAI) in healthy patients and patients with bruxism in tests with pressing in occlusion, grinding and rest. All the values received to hold a high-reliability index, which makes them useful for everyday clinical practice.

Clinical significance: Definition of electromyography (EMG) values impact index of chewing muscles in healthy and bruxing conditions allows to carry out early diagnostics of bruxism and to evaluate the effectiveness of mio relaxation therapy.

Keywords: Bruxism, Electromyography, Masticatory muscles.

How to cite this article: Soykher M, Orlova O, Soykher M, Shershneva D, Vekilyan L, Kotlyarov V, Soykher E, Stroganova A. Electromyography Values of Chewing Muscles in Healthy and Bruxing Conditions. World J Dent 2018;9(5):345-348.

Source of support: Nil

Conflict of interest: None

\section{INTRODUCTION}

For the present day, the phenomenon of bruxism is not fully investigated. The wide range of prevalence of this

\footnotetext{
${ }^{1}$ Institute of Biotechnologies and Interdisciplinary Dentistry, Komsomolsky, Moscow, Russian Federation

${ }^{2}$ Sechenov University, Bolshaya Pirogovskaya, Moscow, Russian Federation

Corresponding Author: Anna Stroganova, Sechenov University, Bolshaya Pirogovskaya, Moscow, Russian Federatio, Phone: +7-967-095-86-34, e-mail: stroganova1711@mail.ru
}

disease (5 to $80 \%$ ) is mostly concerned with deficiency of a common terminology and with patients' ignorance of the occurrence of parafunctional night-time habits). ${ }^{1}$ Bruxism is normally defined as a parafunction that is realized as a pressing and grinding of upper and lower teeth between themselves. During that action, extremely high forces can be developed that exceed forces that occur during chewing. ${ }^{2}$ International Classification of Diseases 10th Revision (ICD-10) attributesbruxism to somatoform disorders (class V, psychic disorders and, F 45.8-Other somatoform disorders). ${ }^{3}$ In the second International Classification of Sleep Disorders (ICSD-2) bruxism is defined as an or motor activity, characterized or grinding of teeth during sleep, and commonly related with the micro-activation reaction on electroencephalogram, and generally associated with sound. ${ }^{4}$ Kato et al. attributedbruxism to parasomnias and parafunctional activity during sleep, characterized by pressing of teeth (tonic activity) and/or recurrence of muscle activity phases (phasic activity). ${ }^{5}$ In 2008, American Academy of Orofacial Pain (AAOP) defined bruxism as a day and night unconscious parafunctional activity, including pressing and grinding of teeth). ${ }^{6}$ We hold on to R Slavicek's and S Sato's theory that considers bruxism as a vital body function, as a precautious resource of prevention of diseases caused by stress. ${ }^{2}$ However, nowadays it is quite hard to value the line when a normal stress-management body function is transformed into a parafunctional activity, damaging the structural components of dentition. Despite the big amount of papers, dedicated to the investigation of the present motive phenomenon, the aspects of its etiology and pathogenesis are still differently interpreted. The multi-factor origin of the disease along with typical peculiarities of patients' personality (high level of anxiety, boundary psychic disorders) are considered generally recognized. ${ }^{7-10}$ Bruxism varies versatile in clinical picture, with the most common features are: hypertrophy of chewing muscles, pain and tiredness of these muscles, especially in the morning, limitation of mouth opening, pathological teeth abrasion, not rarely leading to disease of vertical dimension; teeth marks on the lateral surface of tongue and cheeks, cracks and fractures of teeth crowns and roots. Headaches, irritability, anxiety, and sleep 
disorders are possible. Bruxism frequently has a concealed current and is being diagnosed only during the dental examination. ${ }^{11}$ If the current of the illness was concealed, the presence of bruxism has been discovered by relatives, who pointed on night time gritting of teeth in their households. ${ }^{12,13}$ Because of polyetiological origin of the disease, variable clinical picture a complex examination and participation of doctors of different specialties is necessary, including dentists, neurologists, andpsychiatrists. As for dentists in everyday practice, diagnostics of early stages of the disease is necessary, it has a big impact for treatment planning in patients with an active stage of bruxism, since classic restorations, fillings, prosthodontic constructions are short-lived in such patients. ${ }^{13-15}$

A portable superficial EMG can be a simple method of early diagnosis of bruxism. A proposed diagnostic method is remarkable for its ease of application, noninvasiveness, and innocence for patients, it does not cause discomfort, doesn't demand a long preparation of the doctor and specially equipped premise. The equipment, used for this investigation, is available for the majority of dental clinics. A diagnostic process is, first of all, a process of finding some abnormality. As soon as we have a conception of the norm, we can put a diagnosis, make a correct treatment plan and expect a stable result. But the existing data of electromyographic investigation of chewing muscles has a number of inaccuracies. There are no strict characteristics of normal bioelectric activity of chewing muscles described in the literature. ${ }^{16}$ As a result, superficial EMG data is contradictory and demands further investigation, and the interpretation of the results stays as a privilege for a limited group of specialists.

\section{AIM}

The aim of this study is the definition of EMG values of chewing muscles in healthy and bruxing conditions.

\section{MATERIALS AND METHODS}

Investigation of 68 patients was carried out. Patients with bruxism were divided into two groups: 1st group-15 people, that were treated with type A (Botox) botulinum toxin (group-botox), 2nd group 53 people, not having treatment and with complaints of bruxism in the present time (group bruxism). The control group (group-no complain) consisted of 26 people without complaints on bruxism. The exclusion criteria for the study were: severe psychological disorders and/or the use of antipsychotic drugs, central or peripheral nervous system disorders.

The diagnosis (Bruxism) was put according to the American Academy of Sleep Medicine (AASM) criteria. ${ }^{16}$ The present method of clinical diagnostics grounds depend on two indicators. First of all, the patient himself tells us about the presence of pressing and gritting of teeth, and also the presence of one of the following clinical symptoms: pathologic teeth abrasion or soreness in chewing muscles. Superficial EMG was used for registration of bioelectric action potential. The investigation was held with the use of synapsis (Neurotech, Russia) EMG device. The EMG activity of chewing muscles was registered simultaneously on both sides with the use of a pseudo monopolar method of recording. Three samples were analyzed: pressing of teeth, grinding of teeth and rest, the duration of each sample was 10 seconds. The statistic data reconfiguration was held in a statistic analysis system STATISTICA 8.0. The Levene test, two-sampled t-criteria for independent samples and Wilcoxon signed-rank test were used. Mathematical calculations and digitized data reconfiguration were made using statistical analysis methods with a glance of specialist's recommendations.

\section{RESULTS}

Remarkable differences were found in groups Botox-no complains in tests-rest and pressing in occlusion for CMSI\%. The CMSI in group botox during pressing in occlusion was $74 \%$ and during rest was $102 \%$. The CMSI in the group no complains, for pressing in occlusion was $118 \%$ and for rest was $106 \%(p<0,03)$.

The impact index distribution for groups is described in Table 1.

\section{DISCUSSION}

When comparing of mean values of variables with the use of student $t$-criteria, we discovered that group botox and no complains are homogeneous by the majority of parameters. Therefore, we can assume that type A botulinum toxin therapy is an effective method of muscle relaxation as it brings the majority of the exponents in Botox (treated bruxists) group to normal values. Remarkable differences were found in pares Botox-no complains in rest and pressing in occlusion tests in CMSI\%. CMSI is calculated by the formula as a relation of the lower chewing muscle biopotential amplitude (a minimum) one

Table 1: The values of total bioelectric muscle activity (IMPACT) in different groups.

\begin{tabular}{llll}
\hline Trial & No complain group IMPACT, mcV & Bruxists group IMPACT, mcV & Botox group IMPACT, mcV \\
\hline Pressing in occlusion & $1985 \pm 1344$ & $3649 \pm 1789$ & $2314 \pm 1532$ \\
Grinding & $1238 \pm 696$ & $2404 \pm 1622$ & $1299 \pm 733$ \\
Rest & $299 \pm 61$ & $297 \pm 64$ & $300 \pm 60$ \\
\hline
\end{tabular}


side to the higher chewing muscle biopotential amplitude (a maximum) on the opposite side. CMSI is significantly higher inno complains group than in Botox group, wherein the CMSI did not differ in Bruxists group and no complains group, but it was significantly higher after treatment with Botox. Such a difference in symmetry can be caused by the impact of the botulinum toxin, therefore, we can conclude, that the dosage of the toxin should be held more precisely. There is a dosage calculation method in injections of neuropeptide in patients with bruxism that is based on a medical technology according to skeletal classes. Thus, in patients with skeletal class 1, we used 50 units of the drug to the masseteric muscle and 15 units in temporal muscle. However, each muscle differs from another, and such a standard approach leads to the fact that after botox injection the CMSI was getting even lower, than in bruxing patients with no therapy with neuropeptide. Further investigations should be directed to make a precise calculation of the dosage for each muscle concordant to EMG examination. ${ }^{15}$

In the synapsis device the calculation of different digital EMG analysis wasdone: amplitude, turns, front, maximal and mean phase and turn amplitudes, different index values. Each of these values needs a description and normative chewing muscles values pursuit. After analyzing data, it was found, that square and A mean values were significantly higher in a Pressing in occlusion» test in each channel and every group. Therefore, we can assume, that these values most clearly reflect the total functional activity of chewing units. Their recruitment degree in our opinion is reflected by those values. Thus, under neuropeptide influence having a shut-down of a part of motor units from the active pool, we could see the decreasing of the absolute «Square» and A mean values.

We began the statistical analysis with the valuation of the impact index-a sum of four mean amplitudes of the analyzed muscles.

The variants of the norm of the total bioelectric muscle activity (IMPACT) have different values in total (Table 1). Thus, IMPACT in pressing of teeth test in no complain group is $1985 \pm 1344 \mathrm{mcV}$. Therefore, normal values span from $641 \mathrm{mcV}$ to $3329 \mathrm{mcV}$. In the grinding test in No complains group IMPACT is consistently lower than in pressing of teeth trial. That tells us that static loadings on their own are more dangerous and can have a bigger mechanic influence on the stomatognathic system. By analyzing amplitude, we can not speak about the muscle strength, but we can assume, that the bigger the amplitude-the bigger the strength of the traction. ${ }^{16}$ In the «grinding» test the norm is $1238 \pm 696 \mathrm{mcV}$, and it keeps within $542 \mathrm{mcV}$ to $1934 \mathrm{mcV}$, in the rest test IMPACT is $299 \pm 61 \mathrm{mcV}$ (between the borders form $238 \mathrm{mcV}$ to 360 $\mathrm{mcV}$ ) (Table 1).
The total bioelectric muscle activity in the bruxists group is $3649 \pm 1789 \mathrm{mcV}$ (Table 1). In the grinding test, the IMPACT is $2404 \pm 1622 \mathrm{mcV}$. This value is lower than in pressing of teeth test, but higher than in No complains group. Distinctive values for Bruxists group are that IMPACT in rest trial is $299 \mathrm{mcV}$.

Analyzing the Botox group, we found that all the meaning are very close to the no complains group values. Such a distribution can witness the effectiveness of the myorelaxation of the chewing muscles with the type A botulinum toxin. IMPACT in pressing in occlusion is $2314 \pm$ $1532 \mathrm{mcV}$, in grinding test it is $1299 \pm 733 \mathrm{mcV}$, in the rest trial it is $300 \pm 60 \mathrm{mcV}$ (Table 1$)$.

\section{CONCLUSION}

As a result of the research, we discovered normative values of total bioelectric activity of muscles in three tests: pressing of teeth, grinding of teeth in rest in healthy patients and patients with bruxism. Even with the positive outcomes shown from the study, there are needs for more studies performed on a larger sample size for the narrowing of the intervals of normal distribution (for example IMPACT in "grinding: test in healthy patients is from $542 \mathrm{mcV}$ to $1934 \mathrm{mcV}$ )/ Nevertheless, within the limitation of our study all the findings had a high index of reliability, which makes them useful for everyday clinical practice. Further investigations should be dedicated to the narrowing of the intervals of a normal distribution, description of the parameters of the norm for the remaining EMG values, bruxism biomarkers pursuit and the specification of the dosages of type Abotulinum toxin for each muscle according to EMG examination.

\section{CLINICAL SIGNIFICANCE}

Definition of EMG values IMPACT index of chewing muscles in healthy and bruxing conditions allows to carry out early diagnostics of bruxism and to evaluate the effectiveness of mio relaxation therapy.

\section{REFERENCES}

1. Slavicek R, Sato $S$. Bruxism a function of the masticatory organ to cope with stress. WMW. 2004 Dec;154:584-589.

2. International Classification of Diseases 10th Revision. World Health Organization;2010.

3. International Classification of Sleep Disorders (Revised). Diagnostic and Coding Manual. Rochester: American Sleep Disorders Association;1997

4. Kato T, Thie NM, Huynh N, Miyawaki S, Lavigne GJ. Topical review: sleep bruxism and the role of peripheral sensory influences. J Orofac Pain. 2003 Summer;17:191-213.

5. American Academy of Orofacial Pain. Orofacial pain: guidelines for assessment, diagnosis, and management. 4th ed. Chicago: Quintessence;2008. pp 190-200. 
6. Manfredini D, Lobbezoo F. Role of psychosocial factors in the etiology of bruxism. J Orofac Pain. 2009 Spring;23:153-166.

7. Skorikova LA. Diagnostics, orthopedic treatment of patients with parafunctions of masticatory muscles in treatment of neurotic conditions. PhD thesis. Krasnodar krai, Krasnodar: Kuban State Medical Institute named after the Red Army; Dec.12,1992.

8. Sato S, Slavicek R. The masticatory organ and stress management. J. Stomat. Occ. Med. 2008 Oct 1;1:51-57.

9. Khvatova V. Functional diagnostics and treatment in dentistry. Moscow: Medical book; 2007 Jul 11; pp 294.

10. Seferyan NU. Clinical pattern and comprehensive treatment of parafunctions of chewing, mimic and tongue muscles. PhD thesis. Tver, Tveroblast:Tver State Medical Academy; Dec.11,1998.

11. Virgunova TV. Bruxism in young people: peculiarities of clinical pattern, diagnostics and treatment. PhD thesis. Tver, Tver oblast:Tver State Medical Academy; 2013 Nov 26.
12. Gaydarova TA. Mechanism of generation and pathogeneticprinciples of bruxism treatment. PhD thesis, Irkutsk, Irkutsk oblast:Irkutsk State Medical University; 2003 Dec 26.

13. Dolgalev AA. Personal approach tactics at dental arches repair in patients with TMJ and chewing muscles disfunctions. PhD thesis. Stavropol, Stavropol krai:Stavropol State Medical Academy; 2009 May 19.

14. Ferrario VF, Sforza C, Colombo A. An electromyographic investigation of masticatory muscles symmetry in normoocclusion subjects. J. Oral Rehabil. 2000 Jan;27:33-40.

15. Trezubov VN. Clinical dentistry. Moscow: Practical medicine;2015. pp 76-78.

16. American Academy of Sleep Medicine. International classification of sleep disorders, 2nd ed. Diagnostic and Coding Manual. Westchester. Illinois: American Academy of Sleep Medicine; 2005. 